\title{
Continuous-flow microextraction and gas chromatographic-mass spectrometric determination of polycyclic aromatic hydrocarbon compounds in water
}

\author{
Yan Liu ${ }^{a}$, Yuki Hashi ${ }^{\text {a,b }}$, Jin-Ming Lin ${ }^{\text {a,* }}$ \\ a State Key Laboratory of Environmental Chemistry and Ecotoxicology, Research Center for Eco-Environmental Sciences, \\ Chinese Academy of Sciences, P.O. Box 2871, Beijing 100085, China \\ ${ }^{\mathrm{b}}$ Shimadzu (HongKong) Limited, Beijing Office, Analytical Applications Center, Chao Yang Men Wai Street, Life Tower, \\ Chao Yang District, Beijing 100020, China
}

Received 21 September 2006; received in revised form 30 December 2006; accepted 8 January 2007

Available online 13 January 2007

\begin{abstract}
A new method of the determination polycyclic aromatic hydrocarbons (PAHs) in water samples was developed by continuous-flow microextraction (CFME) coupled with gas chromatography-mass spectrometry (GC-MS). In this experiment, $15 \mathrm{~mL}$ sample solution with no salt-added was flowed at the rate of $1.0 \mathrm{~mL} \mathrm{~min}^{-1}$ through $3 \mu \mathrm{L}$ benzene as extraction solvent. Under the optimal extraction conditions, the developed method was found to yield a linear calibration curve in the concentration range from 0.05 to $15 \mathrm{ng} \mathrm{mL}^{-1}$. Furthermore, the accuracy and repeatability of the method were good by calculating from water samples spiked at known concentrations of PAHs, and the recovery of optimal method was satisfactory. The results showed that CFME was an efficient preconcentration method for extraction of PAHs from spiked water samples.

(C) 2007 Elsevier B.V. All rights reserved.
\end{abstract}

Keywords: Continuous-flow microextraction; Polycyclic aromatic hydrocarbons; Gas chromatography-mass spectrometry; Water analysis

\section{Introduction}

Polycyclic aromatic hydrocarbons (PAHs) are defined to be composed of two or more fused aromatic rings containing only carbon and hydrogen atoms. They are formed mainly by the incomplete combustion of fossil fuels and coal, petrochemical cracking processing and the degradation of lubricating oils and dyes. PAHs are of wide concern because some of them are classified as probable human carcinogens [1] and show tumorigenic activity and endocrine-disrupting activity in mammals [2]. They exist widely in the atmosphere, soil and water and contact tightly with human health. As high efficient separation tools, GC and HPLC have been used widely for separating all kinds of samples containing complex components. However, the direct determination of extremely trace PAHs by modern

\footnotetext{
* Corresponding author. Present address: Department of Chemistry, Tsinghua University, Beijing 100084, China. Tel.: +86 1062792343 ; fax: +861062792343 .

E-mail address: jmlin@mail.tsinghua.edu.cn (J.-M. Lin).
}

high efficient chromatographic techniques, such as GC-MS [3] and HPLC-UV/FL [4], is still very difficult because the content of PAHs is very low, such as ng level or pg level in water [5]. Therefore, preconcentration techniques are often required. Liquid-liquid extraction (LLE) [4], liquid-phase microextraction (LPME) [6], solid-phase extraction (SPE) [7,8], solid-phase microextraction (SPME) [9-12], liquid membrane extraction (LME) $[13,14]$ and cloud-point extraction (CPE) $[15,16]$ have been developed to separate and concentrate the trace level PAHs in the environmental water.

Traditional LLE was used widely due to its higher potential for physical separation by using mixed solvent and a higher capacity for interfering compounds. However, it was substituted gradually by some rapid, cheap, and convenient pretreatment techniques because of its time-consuming nature and usage of large amounts of expensive high-purity organic solvents. Therefore, many attempts to develop LLE procedures include mainly in two aspects, i.e. miniaturization and automation. LME technique provides the possibility of the automation of LLE. Supported LME [17] is based on a three-phase system with an organic phase sandwiched between two aqueous phases and the 
organic phase is immobilized in a porous hydrophobic membrane. Continuous-flow LME [18] was developed in order to extract polar substances and prolong the life span of the liquid membrane, which was very easy to realize the automation with HPLC. LPME [19], using a microliter drop of organic solvent on the end of a Teflon rod or the needle tip of a microsyringe, was developed as a cheap pretreatment method. It has mainly two modes of static LPME [20] and headspace LPME [21]. Drop of organic solvent in static LPME mode immerses directly in the stirred aqueous solution and in headspace LPME mode it is laid above the aqueous solution. More recently, Liu and Lee [22] introduced a method termed as continuousflow microextraction (CFME) in order to improve the mass transfer between organic phase and the aqueous solution. Comparing with LPME, the surface of extraction solvent in CFME is refreshed continually when sample solution flows continuously by a pump from the bottom of a virtually immobilized organic drop located on the tip of a microsyringe, which is beneficial to improve extraction efficiency. CFME was developed based on the LLE as a novel sample preparation technique and was fit for the thermodynamic and kinetic equations of LLE:

$C_{0, \text { eq }}=K C_{\mathrm{aq}, \mathrm{eq}}=K C_{\mathrm{aq}, \mathrm{ini}} /\left(1+K V_{0} / V_{\mathrm{aq}}\right)$

$\frac{\mathrm{d} C_{0}}{\mathrm{~d} t}=A_{\mathrm{i}} \beta / V_{0}\left(K C_{\mathrm{aq}}-C_{0}\right)$

where $C_{0, \mathrm{eq}}$ is the analyte concentration in the organic phase (solvent drop) at extraction equilibrium; $C_{\mathrm{aq}, \text { eq }}$ and $C_{\mathrm{aq} \text {,ini }}$ are the equilibrium and initial analyte concentrations in the aqueous phase, respectively; $V_{0}$ and $V_{\mathrm{aq}}$ are the organic and aqueous phase volumes, respectively; $K$ the distribution coefficient; $C_{0}$ and $C_{\mathrm{aq}}$ are analyte concentrations in the organic and aqueous phases at time $t$, respectively; $A_{\mathrm{i}}$ the interfacial area; $\beta$ the overall mass transfer coefficient with respect to the organic phase. As for the microextraction, the absolute amount of analytes extracted to the solvent during extraction is usually negligible compared with its total amount in solution. Thus, analytes are extracted to the organic solvent as soon as possible and there are high enrichment factor. This method was used to determine the nitroaromatic and chlorobenzenes, and their enrichment factors exceeded 1000fold. This pretreatment method was also applied to the volatile halohydrocarbons [23] and five commonly used pesticides [24] in water samples. Up to the present, there was no report about application of CFME in analysis of PAH in aqueous solution.

In this work, 16 PAHs in water samples were determined by continuous-flow microextraction coupled with GC-MS. Experimental parameters affecting extraction efficiency, such as extraction solvent type and volume, flow-rate of sample solution, sample volume and salt concentration, were investigated and optimized. Furthermore, a microinfusion pump can substitute for the HPLC pump or peristaltic pump, which can minimize the chance of producing the air bubble, make the organic microdrop more stable and increase the volume of the sample solution. The optimal method was used to determine PAHs in real water samples.

\section{Experimental}

\subsection{Reagents}

Reference PAHs (16 compounds, each at $2000 \mu \mathrm{g} \mathrm{mL}^{-1}$ ) were obtained from Supelco (Walton-on-Thames, UK). The solution was diluted with methanol to prepared $10 \mu \mathrm{g} \mathrm{mL}^{-1}$ the stock standard solution standard solution. All solutions were stored and refrigerated at $4{ }^{\circ} \mathrm{C}$. Then, working standard solution was freshly prepared by diluting the mixed standard solution with distilled water to required concentrations. $n$-Hexane, benzene, toluene, dichloromethane were all of HPLC-grade. $n$ Butanol was of analytical grade. All glasswares were cleaned thoroughly by soaking overnight in diluted $\mathrm{HNO}_{3}$ and rinsing with ultra-pure water (Millipore, Bedford, MA, USA) before use.

\subsection{Apparatus}

CFME device was composed of home-made glass chamber ( $\sim 0.2 \mathrm{~mL}$ ), a microinfusion pump (Zhejiang University Medical instruments Co. Ltd., Zhejiang, China), $20 \mathrm{~mL}$ glass syringe (Ningbo He Ping syringe factory, Zhejiang, China) and a $10 \mu \mathrm{L}$ GC microsyringe (SGE Scientific, Sydney, Australia), see Fig. 1. A minimum length of PTFE tube (i.d. $0.5 \mathrm{~mm}$ ) was used for all connections.

A Shimadzu GCMS-QP2010 (Shimadzu, Kyoto, Japan) system equipped with a $30 \mathrm{~m} \times 0.25 \mathrm{~mm}$ i.d. fused-silica capillary column with a $0.25 \mu \mathrm{m}$ Rtx-5 ms coating (5\% phenyl: $95 \%$ dimethylpolysiloxane). The carrier gas helium was maintained at a constant linear velocity of $50.4 \mathrm{~cm} \mathrm{~min}^{-1}$. The injector port temperature was set to $270^{\circ} \mathrm{C}$ in splitless mode with the splitter activated after $1 \mathrm{~min}$. The column was held at $90^{\circ} \mathrm{C}$ for $1 \mathrm{~min}$, increased to $180^{\circ} \mathrm{C}$ at the rate of $8^{\circ} \mathrm{C} \mathrm{min}^{-1}$, then increased to $280^{\circ} \mathrm{C}$ at the rate of $15^{\circ} \mathrm{C} \mathrm{min}^{-1}$ for $15 \mathrm{~min}$. The mass spectrometer was operated in the electron impact ionization (EI) mode with an ion source temperature of $200^{\circ} \mathrm{C}$ and the energy of the electrons was kept at $70 \mathrm{eV}$. The interface temperature was kept at $260^{\circ} \mathrm{C}$. The target com-

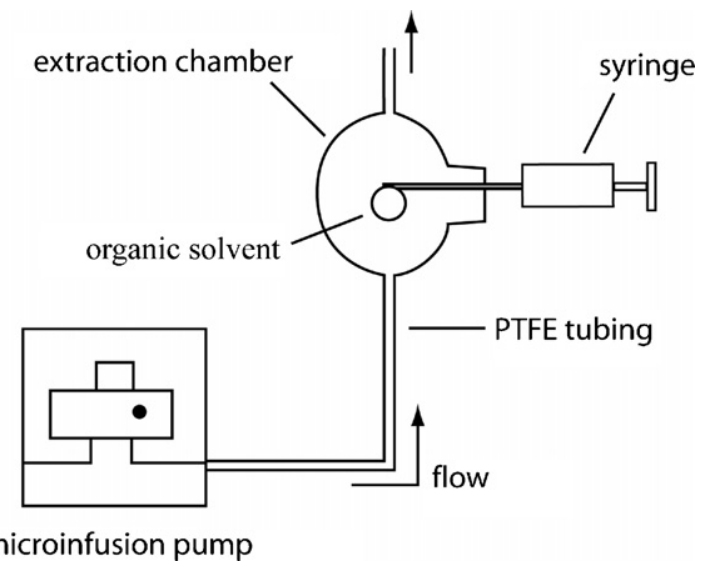

Fig. 1. Schematic diagram of continuous-flow liquid-phase microextraction (CFME). 
pounds were quantified in the selected ion monitoring (SIM) mode, using the molecular ion and one qualifier ions for each compound. The molecular ion and one qualifier ions for naphthalene (NaP), acenaphthylene (Acl), acenaphthene (Ace), fluorene (Fle), phenanthrene (Phe), anthracene (Ant), fluoranthene (Flu), pyrene (Pyr), benz $[a]$ anthracene $(\mathrm{BaA})$, chrysene $(\mathrm{Chr})$, benzo[ $b]$ fluoranthene $(\mathrm{BbF})$, benzo[k]fluoranthene (BkF), benzo $[a]$ pyrene $(\mathrm{BaP})$, indeno[1,2,3-cd]pyrene (IDP), dibenz $[a, h]$ anthracene (DBA) and benzo $[g h i]$ perylene (BghiPe) are 128,$127 ; 152,150 ; 154,153 ; 166,139 ; 178,176 ; 178,176$; 202,$200 ; 202,101 ; 228,226 ; 228,226 ; 252,126 ; 252,250 ; 252$, 250; 276, 138; 278, 276 and 276, 138; respectively.

\subsection{Extraction procedures}

The aqueous sample was infused and filled in the bulb glass extraction chamber via the connecting PTFE tubing. Then $3 \mu \mathrm{L}$ benzene, as the extraction reagent, was introduced into the extraction chamber by $10 \mu \mathrm{L}$ microsyringe, and formed a drop which remained at the tip of the microsyringe. Fifteen milliliters sample solution was flowed continuously through the solvent drop at the rate of $1.0 \mathrm{~mL} \mathrm{~min}^{-1}$. Finally, the solvent drop was retracted into the microsyringe and directly injected into the GC-MS for the analysis.

\section{Results and discussion}

\subsection{Selection of microdrop solvent}

The selection of an appropriate extraction solvent is a key factor for the optimization of CFME process. The extracting solvent needed in this CFMS process should possesses a high distribution coefficient for the analyte and good chromatographic behavior and should be immiscible with water. On the basis of this consideration, $n$-hexane, benzene, toluene, dichloromethane and $n$-butanol were chosen and investigated. From the experimental phenomena obtained, it was found that $n$-butanol was very difficult to hang on the tip of the microsyringe. Dichloromethane and toluene were dissolvable in the sample solvent, resulting in the partly loss of the extracting droplet. Fortunately, benzene and $n$-hexane provided the effective extraction abilities for the studied analytes. The results can be seen in Fig. 2(a) and the extraction ability of benzene was greater than that of $n$-hexane, especially for the analyte containing the benzene ring more than four rings in its structure. Therefore, benzene was chosen and used in the further steps.

\subsection{Microdrop volume}

The effect of drop volume of the extracting solvent was studied in the range of $1-3.5 \mu \mathrm{L}$ and the result was shown in Fig. 2(b). The peak areas of all the analytes increased with the augment of drop volume. When the volume exceeded $3.5 \mu \mathrm{L}$, the drop was not be able to hold on the tip of microsyringe. So $3 \mu \mathrm{L}$ was selected and used in the following experiments.
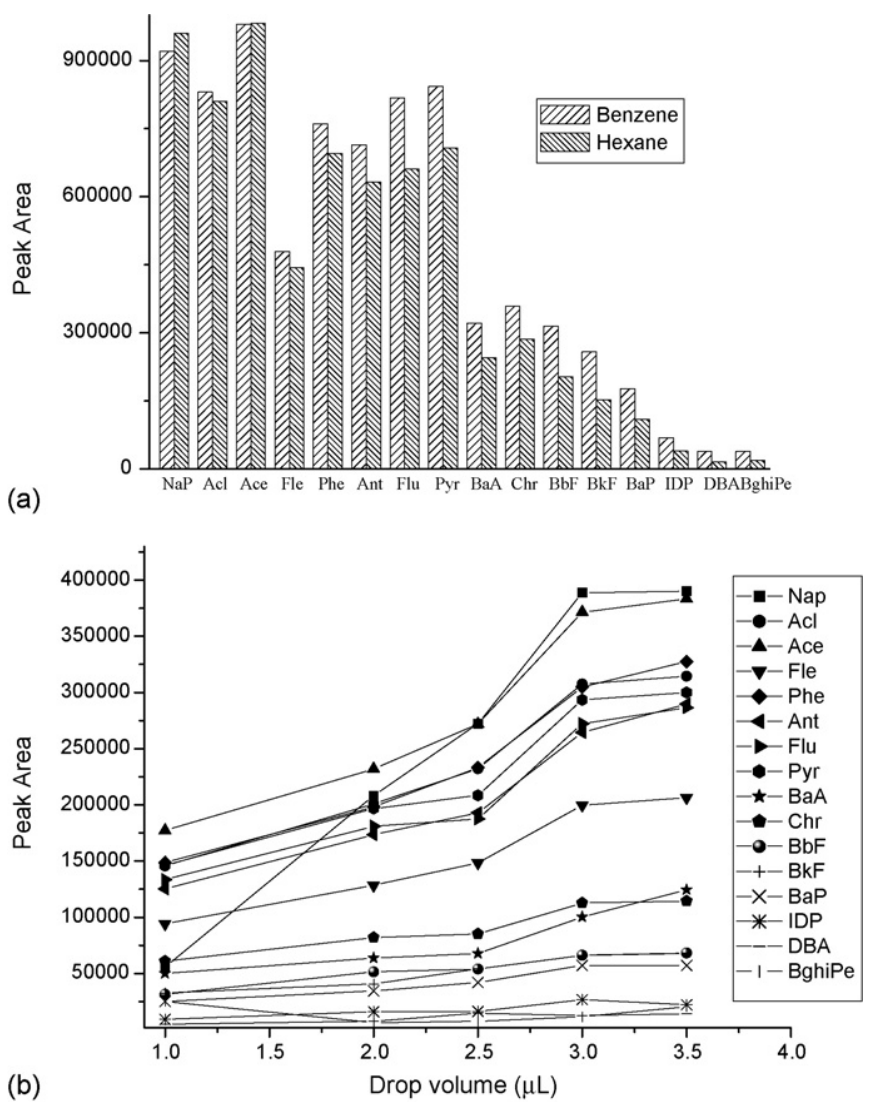

Fig. 2. (a) Influence of solvent type on extraction performance ( $3 \mu \mathrm{L}$ extraction solvent; sample: $12 \mathrm{~mL} 5 \mu \mathrm{g} \mathrm{L}{ }^{-1}$ spiked PAHs in aqueous solution; flow rate: $1.0 \mathrm{~mL} \mathrm{~min}^{-1}$ ). (b) Effect of drop volume on the peak area of PAHs (extraction solvent: benzene; sample: $6 \mathrm{~mL} 5 \mu \mathrm{g} \mathrm{L}^{-1}$ spiked PAHs in aqueous solution; flow rate: $1.0 \mathrm{~mL} \mathrm{~min}^{-1}$ ).

\subsection{Flow rate of sample solution}

Extraction efficiency is normally controlled by the distribution coefficient of the analyte between the aqueous phase and organic phase, which is affected by the thickness of the interfacial layer surrounding the microdrop. The interfacial layer can be varied with the change of the flow rate of sample solution [19]. Therefore, the flow rate of the sample solution in the CFME system needs to be evaluated. The flow rate in the range of $0.5-1.5 \mathrm{ml} / \mathrm{min}$ was considered. It was found that the flow rate of the sample in CFME system played a very significant effect for the extraction ability of the studied PAHs, especially for the low molecular weight analytes. To describe these results, the hydrophobicity and distribution abilities of each analyte should be considered. The obtained results were shown in Fig. 3. At low flow rate $(0.5 \mathrm{~mL} / \mathrm{min})$, the CFME showed low extraction ability. It was due to the low flow rate that provided the wider interfacial layer. The wider interfacial layer might inhibit the distribution of the analyte dissolved in aqueous phase to the organic phase. In addition, at the flow rate higher than $1.0 \mathrm{ml} / \mathrm{min}$, again the extraction ability of the CFME system for the PAHs was decreased. These results might be due to the fact that the extraction equilibrium could not be reached under the very high flow rate condition. Fortunately, the flow rate ranging from 0.8 to 


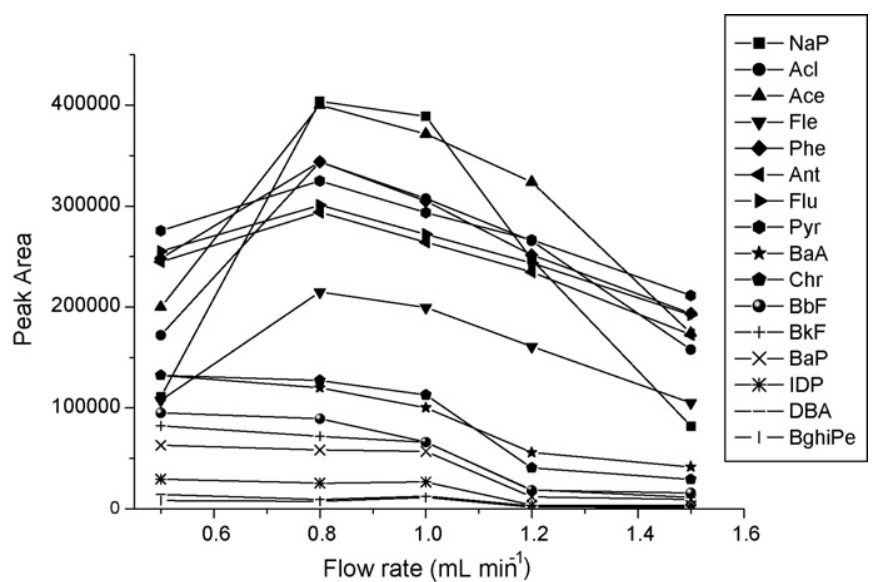

Fig. 3. Effect of flow rate on the peak area of PAHs (extraction solvent: benzene; drop volume: $3 \mu \mathrm{L}$; sample: $6 \mathrm{~mL} 5 \mu \mathrm{g} \mathrm{L}^{-1}$ spiked PAHs in aqueous solution).

$1.0 \mathrm{ml} / \mathrm{min}$ provided the suitable extraction CFME system for PAHs. To compromise between the short sample extraction step and high extraction ability for all analytes, $1.0 \mathrm{ml} / \mathrm{min}$ was eventually chosen and used in the further steps.

\subsection{Sample volume}

In general, the higher enrichment factor could be obtained when higher sample volume is loaded. Importantly, the sample volume passing through the CFME system may seriously affect the loss of the extracting solvent, which would lower the extraction efficiency and lead to the poor sensitivity and precision. Therefore, the sample volume ranging from 5 to $25 \mathrm{~mL}$ was studied and the relationship between sample volume and volume loss of extraction solvent was shown in Fig. 4. The volume loss is equal to the difference between $3 \mu \mathrm{L}$ (initial volume) and the remaining volume of droplet when it is retracted into the microsyringe. When sample solution was $15 \mathrm{~mL}$, the volume loss was just $0.1 \mu \mathrm{L}$. In this condition, there was the best extrac-

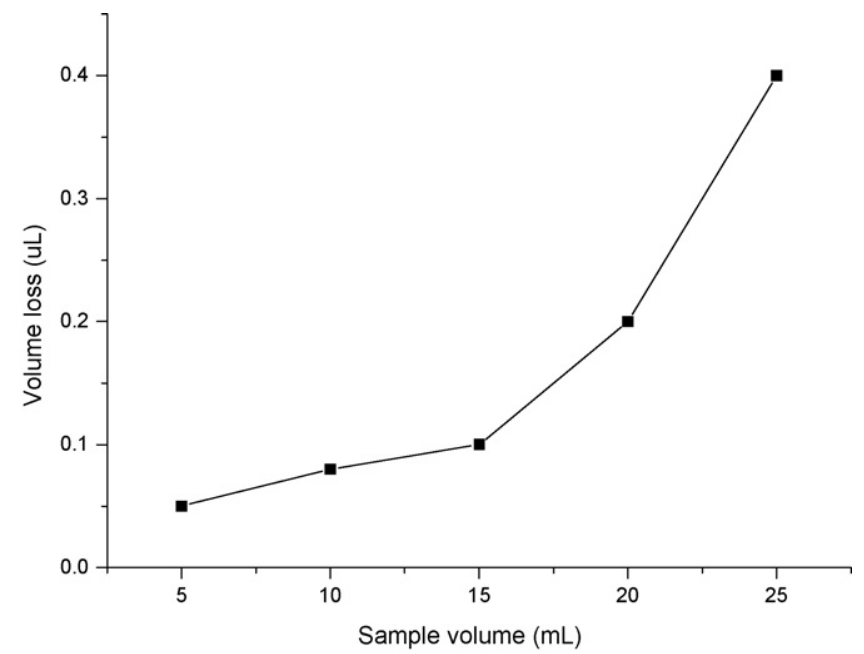

Fig. 4. The relationship between sample volume and the loss of extraction solvent (extraction solvent: benzene; drop volume: $3 \mu \mathrm{L}$; sample: $5 \mu \mathrm{g} \mathrm{L}^{-1}$ spiked PAHs in aqueous solution; flow rate: $1.0 \mathrm{~mL} \mathrm{~min}^{-1}$ ).

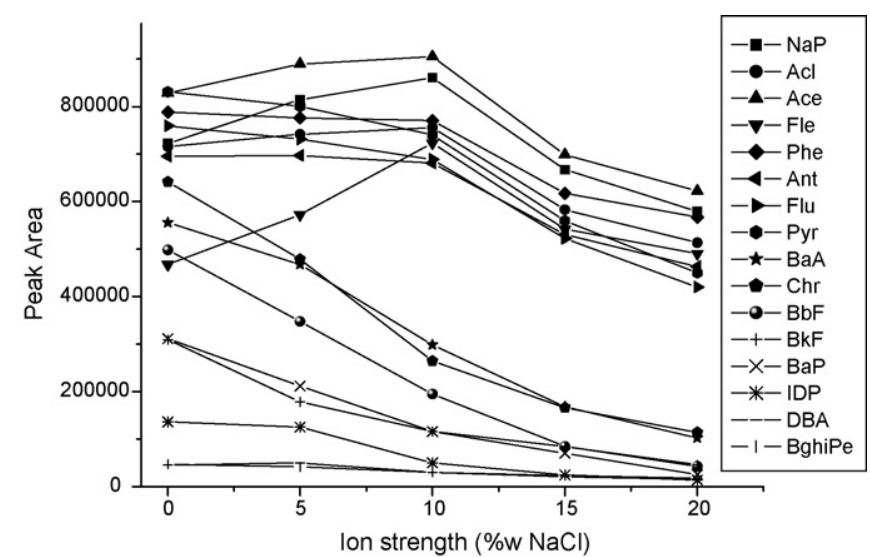

Fig. 5. Effect of ion strength on the extraction performance (extraction solvent:

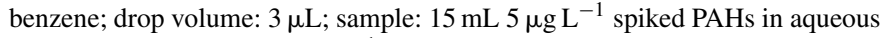
solution; flow rate: $1.0 \mathrm{~mL} \mathrm{~min}^{-1}$ ).

tion efficiency. So $15 \mathrm{~mL}$ of sample volume would be the best choice.

\subsection{Ionic strength}

Generally, the addition of salt to the water causes a decrease in solubility of the organic compounds in water and increases the distribution coefficient, which usually used to enhance the extraction efficiency. Therefore, the influence of salt addition up to $20 \%$ (v/w) on CFME was discussed and the result was shown in Fig. 5. Extraction efficiency for the NaP, Ace and Flu had improved after adding some salt. However, for most PAHs, extraction efficiency had been decreased. It was assumed that apart from the salting-out effect, the presence of salt had changed the physical properties of the extraction film, which reduced the diffusion rates of the analytes into the drop. For the aim of contemporary analysis of $16 \mathrm{PAHs}$, no salt was added in the following experiments.

\subsection{Evaluation of the method performance}

Under the optimum extraction condition, the chromatogram of 16 PAHs in spiked water at concentration of $0.5 \mu \mathrm{g} \mathrm{L}-1$ via CFME was shown in Fig. 6 and some characters of the proposed CFME method such as linear ranges, enrichment factor, correlation coefficients, detection limits and reproducibility were investigated and listed in Table 1. Calibration curves were drawn

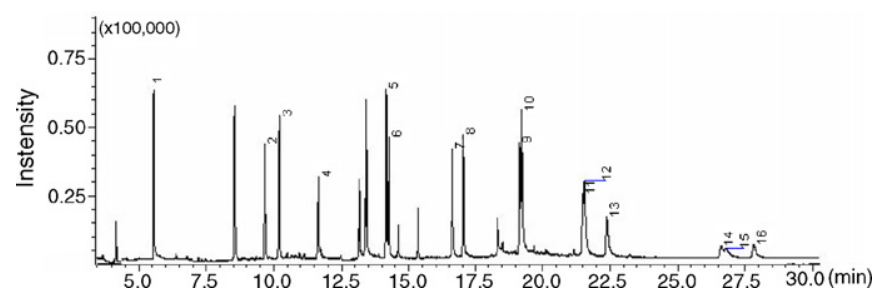

Fig. 6. Chromatogram of $16 \mathrm{PAHs}$ in spiked water at concentration of $0.5 \mu \mathrm{g} \mathrm{L} \mathrm{L}^{-1}$ via CFME under the optimum extraction condition. Peaks: (1) NaP; (2) Acl; (3) Ace; (4) Fle; (5) Phe; (6) Ant; (7) Flu; (8) Pyr; (9) BaA; (10) Chr; (11) BbF; (12) BkF; (13) BaP; (14) IDP; (15) DBA; (16) BghiPe. 
Table 1

Enrichment factor, correlation coefficients, linearity, limit of detections and reproducibility for the proposed CFME method of PAHs

\begin{tabular}{lllllc}
\hline PAHs & $\begin{array}{l}\text { Enrichment } \\
\text { factor }\end{array}$ & $\begin{array}{l}\text { Correlation } \\
\text { coefficient }\left(r^{2}\right)\end{array}$ & $\begin{array}{l}\text { Linear range } \\
\left(\mu \mathrm{L} \mathrm{L}^{-1}\right)\end{array}$ & $\begin{array}{l}\mathrm{LOD}^{\mathrm{b}} \\
\left(\mu \mathrm{L} \mathrm{L}^{-1}\right)\end{array}$ & $\begin{array}{l}\text { R.S.D. } \\
(\%)\end{array}$ \\
\hline $\mathrm{NaP}$ & 83 & 0.9990 & $0.05-15$ & 0.001 & 8.9 \\
$\mathrm{Acl}$ & 87 & 0.9972 & $0.05-15$ & 0.004 & 8.7 \\
$\mathrm{Ace}$ & 79 & 0.9970 & $0.05-15$ & 0.003 & 9.6 \\
$\mathrm{Fle}$ & 82 & 0.9948 & $0.05-15$ & 0.001 & 9.6 \\
$\mathrm{Phe}$ & 75 & 0.9949 & $0.05-15$ & 0.005 & 8.4 \\
$\mathrm{Ant}$ & 78 & 0.9944 & $0.05-15$ & 0.01 & 10 \\
$\mathrm{Flu}$ & 66 & 0.9934 & $0.05-15$ & 0.002 & 8.4 \\
$\mathrm{Pyr}$ & 67 & 0.9941 & $0.05-15$ & 0.002 & 8.9 \\
$\mathrm{BaA}$ & 31 & 0.9941 & $0.05-15$ & 0.002 & 8.9 \\
$\mathrm{Chr}$ & 18 & 0.9948 & $0.05-15$ & 0.002 & 6.7 \\
$\mathrm{BbF}$ & 28 & 0.9947 & $0.1-15$ & 0.006 & 8.4 \\
$\mathrm{BkF}$ & 20 & 0.9234 & $0.1-2.0$ & 0.006 & 8.4 \\
$\mathrm{BaP}$ & 13 & 0.9366 & $0.1-2.0$ & 0.01 & 8.7 \\
$\mathrm{IDP}$ & 9 & 0.9352 & $0.1-2.0$ & 0.007 & 25 \\
$\mathrm{DBA}$ & 3 & 0.8090 & $0.1-2.0$ & 0.01 & 15 \\
$\mathrm{BghiPe}$ & 2 & 0.8860 & $0.1-2.0$ & 0.006 & 11 \\
\hline
\end{tabular}

${ }^{\text {a }}$ Enrichment factor was calculated as the ratio of the final concentration of the analyte in the microdrop and its concentration in the original solution.

${ }^{b}$ LOD: limit of detection $(\mathrm{S} / \mathrm{N}=3)$.

using 6 spiking levels of PAHs in the concentration range of $0.1-2 \mu \mathrm{g} \mathrm{L}^{-1}$ with respect to BkF, BaP, IDP, DBA and BghiPe, and using 11 spiking levels of PAHs in $0.05-15 \mu \mathrm{gL}^{-1}$ for the rest of analytes. As can be seen, higher enrichment factor and good linearity were obtained for less than four rings PAHs with the correlation coefficient $\left(r^{2}\right)$ ranging from 0.9948 to 0.9990 . The precision of this method was calculated according to the continuous extraction of five aqueous solutions spiked at $1 \mu \mathrm{g} \mathrm{L}^{-1}$. For the less than four rings PAHs, their relative standard deviations (R.S.D.) were less than $10 \%$.

Table 2

Recovery of CFME of tapwater sample spiked with PAHs at different concentration levels

\begin{tabular}{|c|c|c|c|c|c|c|}
\hline \multirow[t]{2}{*}{ PAHs } & \multicolumn{2}{|l|}{$0.5 \mu \mathrm{gL}^{-1}$} & \multicolumn{2}{|l|}{$1.0 \mu \mathrm{g} \mathrm{L}^{-1}$} & \multicolumn{2}{|c|}{$5.0 \mu \mathrm{g} \mathrm{L}^{-1}$} \\
\hline & $\begin{array}{l}\text { Recovery } \\
(\%)\end{array}$ & $\begin{array}{l}\text { R.S.D. } \\
(\%)\end{array}$ & $\begin{array}{l}\text { Recovery } \\
(\%)\end{array}$ & $\begin{array}{l}\text { R.S.D. } \\
(\%)\end{array}$ & $\begin{array}{l}\text { Recovery } \\
(\%)\end{array}$ & $\begin{array}{l}\text { R.S.D. } \\
(\%)\end{array}$ \\
\hline $\mathrm{NaP}$ & 107 & 7.0 & 93 & 9.8 & 101 & 0.1 \\
\hline Acl & 101 & 0.8 & 96 & 10 & 98 & 3.3 \\
\hline Ace & 102 & 0.5 & 96 & 10 & 101 & 3.8 \\
\hline Fle & 108 & 1.6 & 95 & 9.2 & 102 & 0.8 \\
\hline Phe & 103 & 9.2 & 94 & 8.8 & 101 & 2.0 \\
\hline Ant & 92 & 5.5 & 90 & 12 & 99 & 2.0 \\
\hline Flu & 95 & 0.8 & 97 & 9.0 & 98 & 0.4 \\
\hline Pyr & 94 & 2.4 & 99 & 12 & 98 & 0.1 \\
\hline $\mathrm{BaA}$ & 89 & 8.5 & 87 & 7.1 & 105 & 5.6 \\
\hline $\mathrm{Chr}$ & 91 & 10 & 90 & 11 & 108 & 9.2 \\
\hline $\mathrm{BbF}$ & 94 & 12 & 93 & 9.2 & 106 & 7.4 \\
\hline $\mathrm{BkF}$ & 92 & 1.8 & 91 & 4.9 & $-^{\mathrm{a}}$ & $-^{\mathrm{a}}$ \\
\hline $\mathrm{BaP}$ & 97 & 10 & 81 & 2.8 & $-^{\mathrm{a}}$ & $-^{\mathrm{a}}$ \\
\hline IDP & 70 & 20 & 93 & 18 & $-^{\mathrm{a}}$ & $-^{\mathrm{a}}$ \\
\hline DBA & 107 & 15 & 115 & 4.9 & $-^{\mathrm{a}}$ & $-^{\mathrm{a}}$ \\
\hline BghiPe & 125 & 18 & 101 & 15 & $-^{\mathrm{a}}$ & $-^{\mathrm{a}}$ \\
\hline
\end{tabular}

${ }^{\text {a }}$ The compound was detected but quantification was not possible because the concentration exceeded the linear range.
Finally, the application of this developed method to the real samples was investigated for different spiked concentration levels. Although the method had the low detection limits, no analytes were detected in the blank tap water. Thus, the proposed method was evaluated by the relative recovery in tap water samples spiked with standard mixtures at three concentration levels at $0.5,1$ and $5 \mu \mathrm{g} \mathrm{L}^{-1}$, respectively. The relative recovery is usually employed instead of absolute recovery, as used in exhaustive extraction procedures [25]. Table 2 shows the results of the three replicates of each spiked water samples obtained by the proposed method. For BkF, BaP, IDP, DBA and BghiPe, their recoveries were not given because water samples spiked with standard mixtures at the concentration of $5 \mu \mathrm{g} \mathrm{L}^{-1}$ were not in their linear range. The result demonstrated that the proposed method was a reliable technique for the determination of trace PAHs in water sample.

\section{Conclusions}

The developed method, combination of continuous-flow liquid-phase microextraction with gas chromatographic-mass spectrometric towards analysis of trace polycyclic aromatic hydrocarbon compounds in water samples was reported for the first time. The method has favorable extraction effect and higher enrichment factor, especially to the less than four rings PAHs. The reliable, rapid and convenient method was applied successfully to determine PAHs in the water sample. Further investigations can focus on the appropriate new extraction solvent to extract more than four rings PAHs to improve the sensitivity and precision of the method.

\section{Acknowledgements}

The authors gratefully acknowledge financial support of the National Natural Science Foundation of China (No. 20437020) and Major Research Program of Chinese Academy of Sciences (KZCX3-SW-432).

\section{References}

[1] IARC, Polynuclear aromatic compounds. Part 1. Chemical, environmental and experimental data, in: IARC Monographs on the Evaluation of the Carcinogenic Risk of Chemicals to Humans, vol. 32, International Agency for Research on Cancer, Lyon, 1983, p. 453.

[2] E. Cavalieri, E. Rogan, Mechanisms of tumor initiation by polycyclic aromatic hydrocarbons in mammals, in: A.H. Neilson (Ed.), The Handbook of Environmental Chemistry, Part J. PAHs and Related Compounds, vol. 3, Springer-Verlag, Berlin, 1998, p. 81.

[3] US Environmental Protection Agency: Compendium method TO-13A: determination of polycyclic aromatic hydrocarbons (PAHs) in ambient air using gas chromatography/mass spectrometry (GC-MS), compendium of methods for the determination of toxic organic compounds in ambient air, second ed., 1999.

[4] International Organization for Standardization (ISO): ISO 17993, Water quality-determination of 15 polycyclic aromatic hydrocarbons (PAH) by HPLC with fluorescence detection after liquid-liquid extraction in water, 2002.

[5] E. Manoli, C. Samara, Trends Anal. Chem. 18 (1999) 417.

[6] S. Shahab, Y. Yadollah, B. Naader, Anal. Chim. Acta 489 (2003) 21.

[7] R.M. Marce, F. Borrull, J. Chromatogr. A 885 (2000) 273. 
[8] E.B. Aziza, K. Anders, A.R. Mohamed, J. Chromatogr. A 1114 (2006) 234.

[9] A. Kabir, C. Hamlet, K.S. Yoo, G.R. Newkome, A. Malik, J. Chromatogr. A 1034 (2004) 1

[10] H. Bagheri, A. Salemi, Chromatographia 59 (2004) 501.

[11] A. Mohammadi, Y. Yamini, N. Alizadeh, J. Chromatogr. A 1063 (2005) 1

[12] A.J. King, J.W. Readman, J.L. Zhou, Anal. Chim. Acta 523 (2004) 259.

[13] L. Zhao, H.K. Lee, Anal. Chem. 74 (2002) 2486.

[14] S. King, J. Meyer, A. Andrews, J. Chromatogr. A 982 (2002) 201.

[15] J.L. Li, B.H. Chen, J. Colloid Interface Sci. 263 (2003) 625

[16] T.I. Sikalos, E.K. Paleologos, Anal. Chem. 77 (2005) 2544.
[17] J.A. Jonsson, L. Mathiasson, Trends Anal. Chem. 18 (1999) 318. [18] J. Liu, J. Chao, G. Jiang, Anal. Chim. Acta 455 (2002) 93.

[19] E. Psillakis, N. Kalogerakis, Trends Anal. Chem. 21 (2002) 53. [20] L. Qian, Y. He, J. Chromatogr. A 1134 (2006) 32.

[21] G. Shen, H.K. Lee, Anal. Chem. 75 (2003) 98.

[22] W. Liu, H.K. Lee, Anal. Chem. 72 (2000) 4462

[23] Y.L. Li, T. Zhang, P. Liang, Anal. Chim. Acta 536 (2005) 245.

[24] Y. He, H.K. Lee, J. Chromatogr. A 1122 (2006) 7.

[25] Y. He, Y. Wang, H.K. Lee, J. Chromatogr. A 874 (2000) 149. 